\title{
Psicologia nas Emergências: uma Nova Prática a Ser Discutida
}

Psychological Emergencies: a New Practice to Be Discussed

Psicología en las Emergencias: una Nueva Práctica a Ser Discutida

Mariana Esteves Paranhos \& Blanca Susana Guevara Werlang

Pontifícia Universidade Católica do Rio Grande do Sul

http://dx.doi.org/10.1590/1982-370301202012 
Resumo: Desastres e emergências são eventos desencadeadores de estresse pela exposição a um perigo imediato à integridade física e emocional das pessoas envolvidas, requerendo assim ações imediatas. A Psicologia foi convidada a compor a resposta a tais contextos, devendo, portanto apropriar-se dos pilares que norteiam as intervenções em emergências, delimitando os objetivos e o conhecimento necessário para tal. O objetivo desta revisão da literatura foi identificar os pressupostos epistemológicos desta prática inovadora a partir da busca em artigos científicos, capítulos de livros e resumos de eventos científicos. Apresenta-se um breve histórico sobre o tema e o panorama atual na America Latina e no Brasil. Discute-se os propósitos da Teoria da Crise, Intervenção em Crise, aliado às ideias postuladas pela Psicologia Positiva. As intervenções calcadas nestes alicerces partem da suposição de que pessoas possuem habilidades para superação de forma positiva dos efeitos da crise, e seu foco é na prevenção do crescimento positivo e da deterioração psicológica, que será facilitada pelo apoio social provido por instituições que potencializem a capacidade autoeficiente dos sobreviventes. A intervenção mais amplamente proposta são os primeiros auxílios emocionais (PAE). A atenção a pessoas que experimentam fatos favoráveis a incapacitações físicas e psicológicas se tornará mais comum, considerando a realidade contemporânea, e a Psicologia deve prover assistência visando fortalecer habilidades de enfrentamento em situações críticas.

Palavras-chave: Intervenção na crise. Emergência. Prevenção. Psicologia Positiva.

Abstract: Disasters and emergencies are stressful situations because of exposure to an imminent danger to the physical and emotional integrity of those involved, requiring immediate action. Psychology was considered to decipher answers to such contexts and therefore must own the pillars that guide interventions in emergency situations, setting goals and specifying the required knowledge. The goal of this literature review was to identify the epistemological assumptions of this innovative practice through a survey of scientific articles, book chapters, and abstracts of scientific events. A brief history on the subject is presented along with the current situation in Latin America and Brazil. The purposes of the Theory of Crisis and Crisis Intervention, allied to the ideas postulated by Positive Psychology, are also discussed. Interventions based on these foundations assume that people have skills to positively overcome the effects of the crisis, and the focus is on prevention of positive growth and psychological distress, which will be facilitated by social support provided by institutions that strengthen the survivors' ability to effectively help themselves. Psychological first aid is a widely proposed intervention. Caring for people who experience situations involving physical and psychological distress will become more common, considering contemporary reality, and Psychology should provide assistance aimed at strengthening coping skills in critical situations.

Keywords: Crisis intervention. Emergency. Prevention. Positive Psychology.

Resumen: Desastres y emergencias son situaciones de gran tensión por su exposición a un peligro inminente para la integridad física y emocional de los involucrados, por lo que requiere una acción inmediata. La Psicología fue invitada a componer la respuesta en tales contextos, y por lo tanto debe tener la propiedad de los pilares que rigen las intervenciones en situaciones de emergencia, la definición de los objetivos y los conocimientos requeridos para hacerlo. El objetivo de esta revisión de la literatura fue identificar los supuestos epistemológicos de esta práctica innovadora a través de la búsqueda de artículos científicos, capítulos de libros y resúmenes de eventos científicos. Primero se presenta una breve historia del tema y la situación actual en América Latina y Brasil. Se analizan los propósitos de la Teoría de la Crisis, Intervención en Crisis, aliado a las ideas postuladas por la Psicología Positiva. Las intervenciones basadas en estos conceptos parten del supuesto de que las personas tienen habilidades para superar de forma positiva los efectos de la crisis y la atención se centra 
en la prevención de un crecimiento positivo y el deterioro psicológico, que será facilitada por el apoyo social próvido por instituciones que fomenten la capacidad autosuficiente de los sobrevivientes. La intervención más ampliamente propuesta es a través de los Primeros Auxilios Psicológicos (PAE). La atención a las personas que sufren hechos favorables a problemas físicos y psicológicos se vuelven más comunes, teniendo en cuenta la realidad contemporánea, y la psicología debe prestar asistencia a fin de fortalecer las habilidades de afrontamiento en situaciones críticas.

Palabras clave: Intervención en las crisis. Emergencia. Prevención. Psicología Positiva.

\section{Introdução}

Nos últimos anos, vem se observando uma série de desastres naturais em nível mundial, deixando, assim, a percepção de que esses eventos parecem ser cada vez mais frequentes e mais devastadores. Considerando a última década, presenciou-se o terremoto no Haiti e no Chile no início de 2010, as inundações na Austrália no final de 2011 e início de 2012, e um dos mais noticiados, no Japão, em março de 2011, em que após um terremoto de grande amplitude, o país foi assolado por um tsunami seguido de uma crise geral ocasionada pela contaminação radioativa decorrente de estragos nas usinas nucleares na região principalmente atingida. Outros fatos que podem ser citados como marcantes para a memória mundial, são o sismo e tsunami ocorridos na Ásia em 2004 e o furacão Katrina nos EUA, em 2005. O que torna comum todos esses acontecimentos é que as consequências destes impactam a todos, pelo grau de destruição que ocasionam e pela sensação de vulnerabilidade despertada diante do lastro de sofrimento humano deixado.

O Brasil não é considerado um país que possui potencial para grandes desastres e catástrofes precipitados pela natureza como furacões, terremotos ou tornados, diferente da realidade de outras nações, que se obrigam a ter programas sólidos de prevenção e de atuação antes, durante e depois do ocorrido. Por este privilégio que o país possui, de não estar no centro de acontecimentos como os citados, cria-se, muitas vezes, a falsa sensação de estar livre destes abalos. Entretanto a realidade não tem se mostrado tão generosa. Evidência disto é que nos últimos anos se presenciou as consequências de quando não se tem um plano consolidado de prevenção e ação para situações emergências, como foi o caso das enchentes e deslizamentos ocorridos, por exemplo, em Santa Catarina em 2008, no Rio de Janeiro em 2009 e 2011, todas deixando inúmeros mortos, desaparecidos e desabrigados. Estes são apenas alguns casos, sabe-se que a esta lista vem aumentando ano a ano. Em se tratando do estado do Rio Grande do Sul, também é clara a necessidade de preocupar-se com o planejamento para situações de risco, conforme as enchentes e enxurradas ocorridas em São Lourenço, interior do estado.

De acordo com o informe mundial sobre desastres de 2010, encabeçado pela Federação Internacional de Sociedades da Cruz Vermelha e do Crescente Vermelho, o risco urbano constitui-se hoje um dos principais desafios do século XXI. Para a Organização, ainda que as Nações Unidas não apresentem cifras precisas, cerca de um milhão de habitantes vivem em locais amontoados, de má qualidade e em bairros informais. Em 2020, a previsão é que se passe ao número de um milhão e quatrocentos habitantes nesta realidade. Aliado a esta situação de precariedade, o risco de desastres por mudanças climáticas aumenta nestas localidades de povoamento desordenado, elevando assim o risco de morte e de perder os meios de subsistência e bens essenciais, como moradia, comprometendo ainda mais o entorno e a prosperidade futura daqueles que sobrevivem. No mesmo informe, indica-se que entre os anos de 2000 e 2008, 50.184 pessoas morreram por eventos sísmicos e as inundações foram os eventos que mais afetaram pessoas, atingindo o número de 99 milhões 
de vítimas. Embora não se saiba quantos indivíduos precisamente viviam em zonas urbanas inadequadas, a Federação estima que seja um número considerável.

No entanto, a lista de tragédias coletivas não se resume aos grandes desastres naturais, ainda que estes tomem uma grande proporção pela velocidade em que as informações são passadas hoje, podendo comover qualquer pessoa, em qualquer lugar do mundo, sem que esta possua envolvimento direto com a população atingida. Infelizmente, situações de emergências e acidentes também fazem parte de um grupo de acontecimentos que são geradores de crise e que podem golpear com intensa violência um grupo de pessoas, uma comunidade, um sistema ou uma nação. Neste sentido, para Gárcia e Gil (2004), se pode propor um continuum de maior ao menor estresse coletivo, estando os desastres e catástrofes em um extremo, passando pelas emergências até chegar aos acidentes, ou seja, situações de menor estresse coletivo. Fernández (2007) faz ainda uma diferenciação quanto aos recursos que cada evento exige, diferenciando-se assim a gravidade destes. As emergências seriam situações que poderiam ser resolvidas com serviços assistenciais locais, tanto de médicos como de resgate. Exemplo disso são os acidentes de tráfico. Os desastres, para os autores, exigem maior infraestrutura para prestar ajuda aos feridos que se encontram em maior quantidade, bem como já existe um grau de destruição em uma área maior, levando também a um custo socioeconômico mais elevado. Já as catástrofes, consideradas os eventos mais graves nesta escala, tratam-se de um desastre massivo, que irá acionar mais recursos humanos e materiais em um esforço coordenado para sanar as necessidades das pessoas envolvidas.

Situações de desastres, catástrofes, emergências ou acidentes podem ser classificados como eventos desencadeadores de estresse, por seu caráter imprevisível e pelo perigo imediato que representam à integridade física e emocional das pessoas envolvidas, requerendo, desta forma, ações imediatas. Todos são fontes de destruição em graus diferentes e causam danos materiais e humanos em diferentes proporções. São tragédias que deflagram a fragilidade do ser humano e, muitas vezes, ocasionam um grande desamparo associado a traumas mais ou menos permanentes para sobreviventes e familiares que perderam seu ente querido, sejam elas provocadas pela natureza ou por pessoas.

Nesta perspectiva, se pode enumerar outros eventos dentro do território brasileiro que o colocam em um patamar de um país que precisa urgentemente se preocupar com as consequências durante e após situações desencadeadoras de intensa crise e potencialmente geradoras de sobrecarga a saúde da sociedade e das pessoas diretamente envolvidas. Dentre elas é possível citar incêndios, sequestros relâmpagos, tráfico de drogas, acidentes de transporte, entre outros. Especificamente os acidentes de transporte, por exemplo, foram, em 2008, a $8^{\circ}$ causa de morte no país, representando 3,5\% do total de 1.066 .842 óbitos registrados no Sistema de Informações sobre Mortalidade (Ministério da Saúde, 2010). Já o tráfico de drogas tornou-se literalmente uma guerra civil travada entre governo e traficantes, em que a população, sem opções, permanece em meio aos ataques iniciados pelos dois lados combatentes. Prova disso foi a situação vivenciada no Rio de Janeiro no final de $2010 \mathrm{em}$ que o exército tomou a frente para a retomada do controle de diversos pontos da cidade que antes eram controlados por traficantes. Outra problemática que também merece atenção é a questão dos desabamentos de construções civis, que, precisamente no início de 2012, atingiram fisicamente, emocionalmente e economicamente uma série de pessoas no Rio de Janeiro. Lamentavelmente é de conhecimento de todos que esta não foi a primeira ocorrência no país. Mais recentemente, é possível citar também a situação ocorrida em janeiro de 2013 no centro do estado do Rio Grande do Sul, na cidade de Santa Maria, onde centenas de jovens perderam suas vidas após um incêndio em uma boate, convertendo-se assim em uma das maiores tragédias do país, levando a discussões importantes sobre a segurança em locais como este.

Neste sentido, sabe-se que, de forma geral, qualquer emergência e desastre é resultado 
de um risco não manejado, ou seja, a probabilidade de que um perigo impacte sobre um sistema socioeconômico com certo nível de vulnerabilidade. Gárcia e Gil (2004) afirmam que a convergência destes dois fatores, ameaça e vulnerabilidade, em um momento e lugar, quando não administrado, gera o acontecimento. Medidas preventivas de percepção do risco são vitais, pois, como refere Gómez (2006), seu desconhecimento ou percepção errônea aprofundam a vulnerabilidade, bem como, quando a situação de emergência ou desastre se concretiza, os planos para lidar com o evento são falhos, os profissionais não se encontram habilitados e pode haver uma maximização dos efeitos causados.

Em se tratando das sequelas deixadas após catástrofes, tradicionalmente, conforme Robles e Medina (2002), a resposta em situações como estas incluem primeiros socorros, provimento de refúgios, alimentos e vestuários. No entanto, nos últimos anos, já é notório a importância e inclusão do apoio psicológico tanto para vítimas envolvidas diretamente na situação, assim como cada vez mais se tem dedicado especial atenção aos trabalhadores e voluntários que atuam nestes eventos.

O olhar para as necessidades psicológicas de pessoas que foram atingidas por eventos traumáticos abriu para a Psicologia um novo campo de atuação e como todo processo que é novo, esta prática também convida os profissionais de saúde mental, em geral, a apropriar-se de seu papel em situações críticas, incluindo todo o contexto e implicações que permeiam estas. Para uma melhor capacitação do profissional que se propõe a atuar nesta área se faz necessário a compreensão dos pilares que norteiam as chamadas intervenções em situações de emergência e catástrofes, delimitando assim os objetivos destas e o conhecimento necessário para tal.

\section{Breve história da Psicologia das Emergências}

A necessidade da Psicologia em situações de emergências e desastres está intimamente relacionada, de acordo com Lomeña (2007) com a descoberta de que pessoas podem manifestar, individualmente ou coletivamente, alterações psicológicas, em decorrência do trauma, físico e/ou emocional, produzido por um evento externo. Estudos e guias de práticas (Kraemer, Wittmann, Jenewein \& Schnyder, 2009; North, Hong, Suris \& Spitznagel, 2008; Organización Panamericana de la Salud, 2004, 2006; Panagioti, Gooding \& Tarrier, 2009) têm demonstrado que a saúde mental de pessoas que passaram por eventos de desastres é fortemente abalada, podendo desenvolver manifestações de estresse agudo, estresse pós-traumático, luto complicado, quadros depressivos, comportamento suicida, condutas violentas, consumo indevido de substâncias psicoativas, entre outros. Kraemer et al. (2009), por exemplo, encontraram entre 342 turistas que foram atingidos direta e indiretamente pelo tsunami de 2004 na Ásia que, quando da avaliação em 2007, 16,8\% preenchiam critérios para TEPT, bem como havia evidências de ansiedade e depressão em $17,8 \%$ e $8,0 \%$, respectivamente. Mais recente ainda é o conhecimento de que quaisquer manifestações, patológicas ou não, serão mediadas por diversos fatores, entre eles a própria natureza do evento, as características de personalidade e a vulnerabilidade individual e social dos sobreviventes (OPAS, 2004, 2006). Todavia, o interesse por este campo de atuação é bastante antigo.

Ainda no século XX, James (1968), em sua obra "Memories and Studies", descreve suas observações sobre o terremoto de São Francisco em 1906. O mesmo aponta dois comportamentos que o impressionaram naquele evento: a rapidez de improvisação para colocar ordem no caos e a atitude positiva e de tranquilidade dos sobreviventes. Lomeña (2007) e Valero (2001) citam o trabalho de Stierlim (1909) com sobreviventes de um acidente em uma mina, também no ano de 1906, e depois em 1908 em um terremoto em Messina, na Itália. Coelho (2006), também fazendo um histórico desta disciplina, refere como a primeira pesquisa científica da área, o estudo de Samuel Prince, em 1920, no Canadá, a partir do desastre marítimo ocasionado pela colisão entre dois navios, um deles carregando explosivos, provocando a um tsunami na Nova Escócia. 
Em 1944, Lindemann publica um importante artigo que serve de alicerce para a intervenção em crise moderna. A investigação, que proporcionou o conhecimento da intervenção psicológica pós-desastre, se baseou no auxílio aos sobreviventes e familiares de vítimas de um grande incêndio no clube noturno Coconut Grove, em Boston (EUA). De acordo com Slaikeu (1996), a descrição dos sintomas psicológicos manifestados por estas pessoas foi o ponto central para o desenvolvimento de aportes sobre o processo de luto e suas etapas até a elaboração da perda. A partir destes dados, Caplan (1964) baseou a teoria da crise e a importância da dissolução positiva ou negativa desta, como ponto de passagem para uma psicopatologia ou não.

Após a segunda guerra, nos EUA, é fundado o Comitê para Estudos sobre Desastres, incentivado pelo governo americano (Lomeña, 2007). Em 1954, é lançado um modelo explicativo do estresse na perspectiva dos desastres, realizando por Manis, descrevendo assim que este seria constituído pelo "evento catastrófico, a resposta psicológica a este e de determinantes intrapsíquicos e situacionais" (Lomeña, 2007, p. 31). Nos anos de 1954, Henry Quarantelli, diretor da Disaster Research Center publica "The Nature na Conditions of Panic" e, baseado nos estudos que vinha desenvolvendo nos EUA, desmistifica a crença de que pessoas diante de uma catástrofe evoluem para um pânico generalizado. Para ele, pessoas, diante destas situações tendem a agregar-se, sentindo-se seguros por estarem inseridos em um grupo (Valero, 2001; Lomeña, 2007).

Na década de 1970, também alguns movimentos foram marcantes para o entendimento cada vez maior das diferentes reações diante de episódios impactantes. Em 1970, a Associação de Psiquiatria Americana publicou um manual de "Primeiros Auxílios Psicológicos em Casos de Catástrofes", contendo nele reações clássicas aos desastres e princípios básicos de atuação (Valero, 2001). Já em 1974, nos EUA, segundo Lomeña (2007), é publicada a primeira Lei de Socorro em Casos de Desastres, frente ao reconhecimento que estes podem ser geradores de transtornos emocionais e mentais de forma crônica e incapacitante. Em 1976, Erikson, a partir da inundação de Bufalo Creek, chama a atenção para as consequências emocionais que são resultantes da desorganização social e física da comunidade atingida, chamando este fenômeno de "segundo desastre".

Em meados dos anos 1980, frente a um terremoto ocorrido na Cidade do México, a Faculdade de Psicologia da Universidade Autônoma do México, inicia um programa de intervenção em crises para prestar apoio, do ponto de vista psicológico, as pessoas envolvidas nos abalos sísmicos. No ano de 1986, o Ministério da Saúde da Colômbia, juntamente com a Organização Pan-Americana de Saúde-OPAS, estabelece um programa de atenção primária em saúde mental para vítimas de desastres (Carvalho \& Borges, 2009; Valero, 2001). Neste mesmo período, na America do Norte, Taylor e Frazer (1982), apontam considerações importantes a respeito do conceito de vítimas, que até o momento eram avaliadas de acordo com as lesões físicas apresentadas. Os autores classificam, então, seis categorias de vítimas, que são avaliadas de acordo com o grau de aproximação e relação com o evento trágico: de primeiro grau seriam as pessoas diretamente envolvidas; de segundo grau são os familiares diretos das vítimas de primeiro; em terceiros grau estariam os profissionais das equipes de resgate; em quarto grau a comunidade ao redor da situação; de quinto grau seriam aquelas pessoas que se envolvem pelo simples conhecimento de que esta ocorrendo ou ocorreu tal evento; e, em último grau, pessoas que estão na localidade afetada por motivos de viagem.

Paralelamente a isso, estudos em relação ao trauma e seu efeito em sobreviventes de guerra vieram acontecendo desde a primeira guerra, conforme descreve Robles e Medina (2002). Estes serviram de base para o surgimento de termos como "fadiga de batalha" ou "neuroses de guerra", amplamente utilizados na segunda guerra, culminando então na categoria "Transtorno de Estresse Pós-Traumático" descrita no DSM-III (American Psychiatric Association, 1980). Em 1957, por exemplo, 
na Europa, o Grupo de Doutores Noruegueses realizaram investigações com sobreviventes de campos de concentração, evidenciando o incremento da mortalidade e morbidade com uma forte associação com o trauma e pouca influência com a personalidade anterior e os investimentos terapêuticos nos anos seguintes a catástrofes (Lomeña, 2007).

A questão do trauma inserida no entendimento de desastres, como bem lembra Coelho (2006), foi influenciada pelo conhecimento da psiquiatria, pontos estes que são importantes e devem ser considerados no pós-evento. No entanto, o que se busca hoje também é a intervenção precoce no que diz respeito a manifestações emocionais de curta duração, auxiliando os indivíduos na minimização destes efeitos e na recuperação de seus recursos psicossociais e trabalhando, assim, de forma preventiva para o desenvolvimento de padecimentos emocionais mais graves. Esta tarefa passa essencialmente por capacitar todos que prestaram cuidado às vítimas, nas mais diferentes instâncias, fazendo com que o cuidado psicológico faça parte de todos os momentos de ação em desastres.

Na America Latina, importantes movimentos vêm ocorrendo desde o início dos anos 2000. Em 2001, em decorrência ao incêndio no mercado popular Mesa Redonda, localizado em Lima (Peru), a Sociedade Peruana em Emergências e Desastres é acionada com o objetivo de trabalhar com a população as informações sobre reações psicológicas normais e esperadas para a ocasião, principalmente no que dizem respeito ao luto. Desta forma, se cria uma linha telefônica chamada de "infosaúde" para o atendimento de psicólogos durante 72 horas após o desastre. A experiência alavancou outros processos, como I Congresso de Psicologia das Emergências e dos Desastres também em Lima em 2002. Na ocasião nasceu a Fundação Latino-Americana de Psicologia das Emergências e dos Desastres - FLAPED, que tem por finalidade a reunião de psicólogos comprometidos com a temática (Carvalho \& Borges, 2009; Molina, 2011). Nos anos seguintes, seguindo o mesmo rumo, outros países como Argentina e Chile criam corporações de iniciativa privada sem fins lucrativos ou organizações não governamentais, objetivando o conhecimento científico e a união de profissionais interessados em desenvolver a área. No Chile, especificamente, é criada a Sociedade Chilena de Psicologia das Emergências e Desastres - SOCHPED (Carvalho \& Borges, 2009; Molina, 2011).

Finalmente, em 2007, após as discussões iniciadas no Primeiro Encontro Internacional de Emergências, organizado pela Sociedade Argentina de Psicologia em Emergências e Desastres, de acordo com Molina (2011), e no II Congresso da União Latino-Americana de Psicologia (ULAPSI), ocorrido em Havana, Cuba, foi formulada a declaração de Princípios da nova Rede Latino-Americana de Psicologia em Emergências e Desastres, subscrevendo-se várias entidades de países da America Latina. O objetivo de tal movimento, de acordo com o mesmo autor, foi incentivar o desenvolvimento e o papel da Psicologia em situações de emergências.

No Brasil, conforme Carvalho e Borges (2009), o primeiro registro do processo histórico de inserção da Psicologia em situações de emergências e desastres foi em 1987, com o acidente do Césio-137, em Goiânia, auxiliando a comunidade afetada. No entanto, no decorrer dos anos, quase nada avançou para o crescimento e consolidação da área. Somente nos últimos anos percebe-se a iniciativa para união de esforços para articulação da presença da Psicologia de maneira mais efetiva em situações emergenciais. Em 2006, em Brasília, por iniciativa do Conselho Federal de Psicologia, que já vinha alavancando e participando das iniciativas para organização da categoria no cenário latino americano, e em parceria com a Secretaria Nacional de Defesa Civil, foi realizado o I Seminário Nacional de Psicologia das Emergências e dos Desastres. No mesmo encontro, deu-se a $1^{\text {a }}$ Reunião Internacional por uma Formação Especializada em Psicologia das Emergências e Desastres, elencando então o que deve compor a formação dos futuros profissionais na área (Anais, 2006). 
Assim, norteados pelo CFP, o Conselho Regional de Psicologia da $12^{a}$ Região - SC, em 2008, assinou o termo de Cooperação com a Secretaria Executiva de Justiça e Cidadania do Estado de Santa Catarina, em que estão propostas ações a serem desenvolvidas em conjunto com a Defesa Civil deste estado. Em março de 2011, o Conselho Regional de Psicologia da $6^{\circ}$ região - SP protagonizou oficinas de práticas de Psicologia em Emergências e Desastres em algumas cidades de São Paulo, bem como criou um Grupo de Trabalho de Emergências e Desastres (GTED).

E em novembro do mesmo ano, Brasília sediou mais uma vez o Seminário Nacional de Psicologia das Emergências e dos Desastres, na sua segunda edição, também promovido pelo Conselho Federal de Psicologia. Naquela ocasião foi realizada uma reunião entre os psicólogos presentes e que assistiam ao evento por videoconferência para a constituição de um ator social relacionado à Psicologia em emergências e desastres. Encerrou-se o encontro com a proposta de criação de uma Associação Brasileira de Psicologia nas Emergências e Desastres (ABRAPEDE), que começa a dar os primeiros passos para sua concretude no ano seguinte. Em setembro de 2012, durante a $2^{\text {a }}$ Mostra Nacional de Práticas em Psicologia, realizada em São Paulo, é formalizada, através de uma Assembleia Geral, a fundação da ABRAPEDE com a eleição de sua diretoria e conselho fiscal.

Apesar de ainda serem recentes as iniciativas no país de aproximação com a Psicologia nas emergências, esteéum campo fértil que merece ser cuidado pela categoria. Neste sentido se faz iminente o conhecimento de bases teóricas que sustentem a prática proposta, auxiliando desta forma na criação de uma plataforma mais sólida para a formação de psicólogos para a área de emergências e catástrofes.

\section{Teoria da Crise e Intervenções em Crise}

Ainda que não se tenham teorias ou modelos unificadores para este campo da Psicologia, é possível refletir, a partir de seu objeto de estudo, sobre a visão de homem que esta prática traz consigo, assim como seus pressupostos epistemológicos. Neste sentido, Lorente (2003) define Psicologia das emergências como o "campo psicológico que abarca o conjunto da emergência" (p. 32) o qual compreende a circunstância (a própria emergência atendida), o curso temporal (antes, durante e depois do evento) e os sujeitos implicados (as vítimas, os intervencionistas e as organizações que se inserem).

Assim, a intervenção em situações trágicas, como as já citadas anteriormente, visa, sobretudo, a busca por auxiliar o sujeito (vítimas e/ou intervencionistas) em sua reorganização psíquica e social, com o intuito de minimizar possíveis agravos da saúde, física e emocional. Para isso, se utiliza da ideia que pessoas que sofrem um abalo em sua estrutura, ocasionado por um evento interno ou externo, entram em uma situação denominada crise que, de acordo com Slaikeu (1996, p. 16), é "um estado temporal de transtorno e desorganização, caracterizado principalmente por uma incapacidade do indivíduo para manejar situações particulares utilizando métodos comumente conhecidos para a solução de problemas, e pelo potencial para obter um resultado radicalmente positivo ou negativo". Desta forma, o ponto chave de qualquer intervenção psicológica em um momento de crise, que pode ser decorrente de um acontecimento catastrófico, está calcado no conhecimento de que a pessoa possui habilidades e condições de superação de forma positiva do estresse desencadeador e que a intervenção possui seu foco na prevenção para que o caminho já disponível ocorra da melhor forma possível.

Este entendimento encontra eco na proposta científica que vem sendo desenvolvida desde 1998, por Martin Seligman - a Psicologia Positiva (Seligman \& Csikszentmihalyi, 2000). Tal conjectura faz uma tensão com os modelos mais tradicionais da Psicologia, centrados em sua maioria apenas na intervenção em patologias instauradas. Esta abordagem, de acordo com alguns autores (Paludo \& Koller, 2007; Snyder \& Lopez, 2009), faz um convite a se pensar nas potencialidades do ser humano, 
apropriando-se para isso das condições e processos que fazem a diferença para a saúde deste e não só naqueles que contribuem para o seu adoecimento.

Os mesmos autores ressaltam que estas ideias já estavam presentes nos textos de Maslow (1954) e Rogers $(1959,1961)$, porém, como bem argumentam, tais pensamentos não tiveram o apelo científico necessário para se consolidarem. Da mesma forma, a Teoria da Crise, conforme Slaikeu (1996), possui influência teórica nestes pensadores, enfatizando a realização e o crescimento positivo do homem. O autor acrescenta, ainda, como marco para esta teoria o Darwinismo, levando em consideração o ponto de vista da adaptação da comunidade humana ao seu meio, as ideias Freudianas de que todos buscam sempre a redução da tensão e o estado de plenitude, o enfoque de Erikson e as etapas do desenvolvimento normal e as tarefas de cada uma delas e, por fim, o entendimento de crise teria recebido influência de dados empíricos sobre como os indivíduos lidam com situações altamente estressantes.

Esta última contribuição origina-se do entendimento das manifestações psicológicas negativas provenientes de acontecimentos carregados de sofrimento e fortemente das pesquisas advindas da Segunda Guerra e as repercussões nos sobreviventes. Seligman (2002) aponta justamente este período da história como ponto importante no desenvolvimento da vertente centrada na questão patológica do ser humano, como uma necessidade de dar conta dos sintomas e danos psíquicos apresentados pelos veteranos de guerra. E esta é outra questão a ser considerada no caso das emergências e catástrofes na atualidade - a questão do trauma. Certamente ele deve ser considerado, porém não pode ser encarado como a única possibilidade por quem enfrenta um desastre. Pelo contrário, estas situações ocorrem quando o desfecho é negativo, e não são a maioria.

De fato, a resolução positiva ou negativa, depende da gravidade do evento que a precipitou, e em se tratando de desastres, estes possuem um potencial alto para gerar estresse e ansiedade em um grau demasiado para quem o vive. Entretanto, como Caplan (1964) - precursor da Teoria da Crise - define, a resolução também receberá influência dos recursos pessoais do sujeito e dos recursos sociais que estão disponíveis. E aqui, nos dois últimos tópicos, o psicólogo deve se centrar, ou seja, no olhar para o que o sujeito tem de competências individuais para o manejo da situação e que recursos podem ser ativados para o contorno desta. A psiquiatria preventiva defendida pelo autor enfoca a intervenção precoce para precaver o crescimento positivo e minimizar a deterioração psicológica, encarando assim a crise sempre como um ponto de mudança. Para ele, a questão da crise sempre será transformadora, não sendo possível sair da mesma forma depois de sua vivência. Tais pressupostos vão ao encontro do que Slaikeu (1996) menciona em sua obra: o ideograma chinês para a palavra crise (weji) é composto de dois caracteres, um que significa perigo e outro que significa oportunidade.

Paludo e Koller (2007), com base nos apontamentos de Ryff e Singer (2003), destacam que diante de contextos em que uma mudança significativa é exigida, e se esta é carregada de risco e adversidade, o funcionamento positivo do indivíduo é identificado mais facilmente. Isto se deve também ao fenômeno denominado resiliência, que, de acordo com Yunes (2003), trata-se da habilidade de superar infortúnios, o que implica ser atingido por algo e ser capaz de sair disto de maneira fortalecida. A sua análise, para Ryff e Singer (2003, apud Paludo \& Koller, 2007), permite o entendimento das fortalezas do ser humano, ao invés apenas de suas vulnerabilidades. A Psicologia positiva aliada à compreensão de crise traz então a possibilidade de pensar que, mesmo diante de eventos trágicos, que levam a uma grande tristeza e dor, sempre há a possibilidade de estes serem também geradores de perspectiva e esperança.

Em se tratando de grandes calamidades, os movimentos em prol da reorganização do lugar afetado e o desejo genuíno de muitas pessoas de ajudar nestas situações, mesmo, muitas vezes, tendo perdido todas as suas referências, é incrível. Também os trabalhos 
voluntários que se articulam rapidamente frente à tragédia e à sensibilização da população nas doações são exemplos da atitude positiva que o ser humano é capaz de colocar em prática. Desta forma, o papel do psicólogo faz a diferença, encorajando e facilitando para que as pessoas não sejam somente ajudadas, mas que possam se ajudar, e para não vitimizá-las cada vez mais, convidando-as a um papel ativo neste processo. A principal atenção humana que a psicologia pode oferecer é o conhecimento do potencial humano.

Tal entendimento chama a atenção para a importância que o entorno exerce para a facilitação do processo de resiliência e a manifestação do potencial positivo da crise. De acordo com McNally, Bryant e Ehlers (2003), proporcionar um ambiente seguro, no sentido da proteção a estímulos ameaçadores, atender as necessidades básicas e, acima de tudo, facilitar o apoio social, de preferência contando com a rede social natural das pessoas, são fatores protetores contra o aparecimento de patologias relacionadas ao trauma. Mesmo assim, o comportamento de todas as pessoas envolvidas na resposta a um evento de emergência, bem como o papel das instituições arroladas para a assistência têm forte influência na possibilidade de uma resposta positiva satisfatória para toda a comunidade que sofre com a situação. Neste sentido, Dunmore, Clark e Ehlers (2001) assinalam que a percepção de interações sociais negativas com outros após a vivência de uma situação traumática pode atuar como preditor para o TEPT crônico em maior medida do que a falta de qualquer apoio positivo percebido.

Portanto, a intervenção em crise é um procedimento para exercer influência no funcionamento psicológico do indivíduo durante o período de desequilíbrio, aliviando o impacto direto do evento traumático. O objetivo é ajudar a acionar a parte saudável preservada da pessoa, assim como seus recursos sociais, enfrentando de maneira adaptativa os efeitos do estresse. Nessa oportunidade, devem-se facilitar as condições necessárias para que se estabeleça na pessoa, por sua própria ação, um novo modo de funcionamento psicológico, interpessoal e social, diante da nova situação (Fernández \& Rodrígues, 2002; Wainrib \& Bloch, 2000). Esta intervenção, em situações de emergências e desastres, é baseada nos primeiros auxílios psicológicos (PAP) que, para Slaikeu (1996), têm como metas proporcionar apoio, reduzir o perigo de morte e auxiliar a pessoa com as fontes de ajuda disponíveis. A proposta deste tipo de intervenção é ainda recomendada por importantes organismos como a Organização Mundial da Saúde - WHO e por grupos de peritos internacionais, entre eles a Inter-Agency Standing Committee (IASC) e o Sphere Project (World Health Organization, 2011).

De acordo com o guia da IASC sobre saúde mental e apoio psicossocial em emergências humanitárias e catástrofes (Inter-Agency Standing Committee/United Nations General, 2007), e com o informe da Organização Mundial da Saúde-OMS sobre os primeiros cuidados emocionais (WHO, 2011), os PAP são uma resposta humanitária de apoio a seres humanos que se encontram em sofrimento e precisam de apoio, envolvendo os temas de prestação de cuidado prático sem ser intrusivo, de avaliação de preocupações atendendo as necessidades mais básicas, de conforto e conexão a fontes de informação seguras e apoio social disponível, e, sobretudo, proteção aos afetados no sentido de evitar maiores danos. A OMS determina três princípios básicos de ação da referida intervenção: olhar, ouvir e vincular. Estes princípios de ação orientam os intervencionistas a como verificar a segurança de si e dos envolvidos, como se aproximar às pessoas afetadas e compreender suas necessidades, e como ligá-los com apoio prático e informação (WHO, 2011).

Existem vários modelos de protocolos para aplicação dos PAP (Martín \& Muñoz, 2009) e ainda que possam possuir algumas diferenças quanto aos passos a ser seguidos, todos buscam precocemente e de maneira eficiente proporcionar a autonomia da pessoa para lidar com o acontecimento e retomar o controle de si e do efeito do entorno sobre si. O modelo proposto por 
Salikeu (1996), por exemplo, propõe cinco passos: realizar contato, examinar dimensões do problema, analisar possíveis soluções, assistir à execuções de ações concretas e seguimento. Já Martín e Muñoz (2009) referem sobre o protocolo ACERCARSE, utilizado na Espanha durante os atentados terroristas de 11 de março de 2004. Este trata do ambiente, contato, avaliação, restabelecimento emocional, compreensão da crise, ativar, recuperação do funcionamento e seguimento. A National Child Traumatic Stress Network e National Center for Posttraumatic Stress Disorder (2006) preconiza como ações básicas do núcleo dos PAP o contato e engajamento, segurança e conforto, estabilizar (se necessário), coleta de informações (necessidades atuais e preocupações), assistência prática, conexão com apoios sociais, informações sobre como lidar e articulação com serviços de colaboração. E, no informe da OPAS de 2010, é enfatizado os passos de realizar contato, analisar o problema, analisar as possíveis soluções, executar ações concretas, dar seguimento, estimulação e dar informação. No entanto, é necessário mencionar que a utilização de protocolos, ainda que seja interessante pois orienta e baliza as ações, deve estar articulada com os planos de contingência traçados e debatidos com a comunidade. Estes devem ser moldados de acordo com a realidade a que se pretende empregar, para que não se torne algo impositivo de alguns profissionais e que não cumpre com seu objetivo primordial - ajudar da melhor forma a quem precisa.

É importante diferenciar que os PAP não pretende ser um aconselhamento profissional, e que ainda que convide o sujeito a falar sobre o ocorrido, não implica necessariamente a discussão detalhada sobre o evento em si ou sua análise. Não deve jamais haver uma pressão para que se fale dos sentimentos e reações diante da situação. Assim, não se constituem também em uma forma de terapia, por isso não é um procedimento exclusivo de profissionais de saúde mental, pelo contrário, qualquer intervencionista de emergências deveria estar capacitado para tal, levando em consideração a questão da comunicação com afetados como ponto essencial para uma franca prevenção dos efeitos negativos para o desfecho da crise. Tampouco é um tipo de intervenção que só possa ser oferecida por profissionais, pode ser realizada pelos próprios membros da comunidade. Neste sentido, cabe mencionar que tal capacitação pode ser oferecida por profissionais da Psicologia, ainda que não exclusivamente, que devem se inserir no contexto enunciado como mais um recurso humano integrante no sistema de coordenação e gestão, avaliando quando, como e que tipo de intervenção pode ser necessária em cada caso. Trata-se de desempenhar um papel de assistente, principalmente aos profissionais que ali trabalham. Sua contribuição é no olhar para a subjetividade em meio a uma situação, muitas vezes, caótica e não como detentor das intervenções de cunho psicológico ou como um especialista em patologia.

Outro ponto fundamental para a aplicabilidade dos Primeiros Auxílios Psicológicos é o respeito à cultura e crenças dos locais e pessoas com quem o trabalho ocorrerá. Sendo assim, qualquer protocolo de atuação deve se adaptar as normas sociais e culturais para o contexto que se insere.

Para muitos profissionais de saúde, o dano psicológico associado a uma experiência de desastre, como bem lembra Rubio (2011), é identificado por meios de sintomas que indiquem a presença de algum transtorno, como o Transtorno de Estresse Agudo ou Transtorno de Estresse Pós-Traumático, assim mesmo não se deve perder o propósito de trabalhar com a saúde e com a parte preservada desta e não apenas com a doença. A intervenção em crise, baseada nos PAP, busca este objetivo, sem desconsiderar que algumas pessoas, mesmo com toda a ajuda fornecida, podem vir a desenvolver dificuldades relativas à experiência que, sem dúvida, é traumática. No entanto, a clareza é que no momento do evento, ainda não se pode prever quem pode adoecer e o trabalho deve ser focado para evitar este desfecho. Mais ainda, como a WHO (2011) afirma, todas as pessoas possuem pontos fortes e habilidade para lidar com os mais diferentes desafios da vida. 
A atenção a pessoas que experimentam fatos inesperados e altamente favoráveis a incapacitações físicas e psicológicas será uma atividade cada vez mais comum na sociedade contemporânea, considerando as atuais mudanças climáticas, o crescimento populacional e de cidades, e, sobretudo, a violência a que se assiste constantemente nas histórias de acidentes de carro, homicídios, suicídios, sequestros, etc. Porém, como bem expõe Llinàs (2010), é necessário avaliar e cuidar para não "patologizar" toda e qualquer situação que tenha o potencial para um trauma, impondo desta forma a presença do psicólogo como imprescindível a qualquer vítima e/ou equipe de socorro, policial e voluntários. Como define o autor, a atenção psicológica é mais um componente, evidentemente importante, mas não imperativo, dentro de um sistema com mais funções. Mesmo assim, o psicólogo que se disponibiliza a estar inserido nestes ambientes e prestando auxílio a qualquer pessoa, deve buscar preparo e formação, o que inclui teoria e prática destas vivencias, por exemplo, através de simulados, que servem para treinamento e reciclagem de conhecimentos.

\section{Considerações Finais}

A Psicologia deve ser capaz de prover um campo fértil para que o ser humano, mesmo diante de situações tão complexas e devastadoras, seja capaz de colocar em marcha sua potencialidade de "dar a volta por cima", presente no psiquismo da maioria das pessoas. Trata-se de identificar e fortalecer uma estrutura que já está pronta, apenas se encontra abalada. Para isso, é essencial a preocupação com o entendimento dos fatores que geram o crescimento e desenvolvimento mesmo diante de situações tão adversas como são os desastres e catástrofes.

É vital também que se trabalhe mais em termos de pesquisas empíricas que enfoquem os aspectos positivos e criativos do ser humano nestes eventos, e não apenas a questão do trauma como patologia. O trabalho deve ser para a promoção da saúde de forma plena e não apenas empenhando-se em um aspecto das manifestações psicológicas em situações de emergência como o adoecimento psíquico.

Por fim, cabe ainda aos psicólogos, uma melhor interação com a área e a busca por formação nesta. Isto inclui uma aproximação com conceitos de crise, intervenções em crise, resiliência, gerenciamento de desastres, entre outros. Sempre que um novo campo surge, é importante o debate sobre o papel da Psicologia, suas ferramentas e capacitação dos profissionais psicólogos, para que desta forma aconteça a consolidação de sua prática de forma responsável e adequada. 


\section{Mariana Esteves Paranhos}

Doutora em Psicologia pela Pontifícia Universidade Católica do Rio Grande do Sul, Porto Alegre - RS. Brasil.

E-mail: marieparanhos@gmail.com

\section{Blanca Susana Guevara Werlang}

Doutora em Saúde Mental pela Universidade Estadual de Campinas, Campinas - SP. Docente da Pontifícia Universidade Católica do Rio Grande do Sul, Porto Alegre - RS. Brasil.

\section{Endereço para envio de correspondência:}

Av. Ipiranga, 6690, Sala 228.4. Jardim Botânico. CEP: 90610-000. Porto Alegre - RS. Brasil.

Recebido 10/08/2012, Reformulação 16/01/2014, Aprovado 08/04/2015. 


\section{Referências}

American Psychiatric Association [APA]. (1980). Diagnostic and statistical manual of mental disorders (3a ed.). Washigton, DC: American Psychiatric Association.

Anais. (2006). Seminário Nacional de Psicologia das Emergências e dos Desastres: contribuições para a construção de comunidades mais seguras. Brasília, DF.

Caplan, G. (1964). Principles of preventive psychiatry. New York, NY: Basic Books.

Carvalho, A. C., \& Borges, I. (2009). A trajetória histórica eas possíveis práticas de intervenção do psicólogo frente às emergências e os desastres. In: Anais do 50 Seminário Internacional de Defesa Civil - DEFENCIL. Recuperado em 11 jul. 2012, de http://www. defesacivil.uff.br/defencil_5/Artigo_Anais_ Eletronicos_Defencil_29.pdf.

Coelho, A. L. (2006). Psicologia das emergências e dos desastres: uma área em construção. In: Anais do 1. Seminário Nacional de Psicologia dasEmergênciasedosDesastres:contribuições para a construção de comunidades mais seguras (pp. 62-66). Brasília, DF.

Dunmore, E., Clark, D. M., \& Ehlers, A. (2001). A prospective investigation of the role of cognitive factors in persistent Posttraumatic Stress Disorder (PTSD) after physical and sexual assault. Behaviour Research and Therapy, 39(9), 1063-1084.

Erikson, K. T. (1976). Loss of communality at Buffalo Creek. American Journal of Psychiatric, 133, 302-305.

Federación Internacional de Sociedades de la Cruz Roja y de la Media Luna Roja (2010). Informe mundial sobre desastres 2010. Recuperado em 6 de julho de 2012, de http:// www.ifrc.org/Global/Publications/disasters/ WDR/wdr2010/WDR2010-summary-SP.pdf

Fernández, J. M. (2007). Introducción. In: J. M. Fernández.(Eds.), Apoyo psicológico en situaciones de emergencias (pp. 19-27). Madri: Psicología Pirámide.

Fernández, A. L., \& Rodríguez, B. V. (2002). Intervención en Crisis. Madri: Editorial Sintesis.

Gárcia, M., \& Gil, J. M. (2004). Aproximación conceptual al desastre. Cuadernos de crisis, $3(1), 7-20$
Gómez, C. (2006). Saúde mental na gestão dos desastres: intervenções no cotidiano e nos eventos. In: Anais do 1. Seminário Nacional de Psicologia das Emergências e dos Desastres: contribuições para a construção de comunidades mais seguras (pp. 72-76). Brasília, DF.

Inter-Agency Standing Committee/United Nations General [IASC]. (2007). IASC Guidelines on mental health and psychosocial support in emergency settings. Genebra: Inter-Agency Standing Committee/United Nations General.

James, W. (1968). Memories and studies. New York, NY: Greenwood Press.

Kraemer, B., Wittmann, L., Jenewein, J., \& Schnyder, U. (2009). 2004 Tsunami: long-term psychological consequences for Swiss tourists in the area at the time of the disaster. Australian and New Zeland Journal of Psychiatry, 43(5), 420-5.

Lindemann, E. (1944). Symptomology and management of acute grief. American Journal of Psychiatry, 101, 141-148.

Llinàs, D. R. (2010). Reflexiones sobre la atención humana a las víctimas en las emergencias. Cuadernos de Crisis, 9(1), 19-24.

Lomeña, E. (2007). Breve histórico de la psicología de catástrofes. In J. M. Fernández (Ed.), Apoyo psicológico en situaciones de emergencias (pp. 29-41). Madri: Psicología Pirámide.

Lorente, F. (2003). Reflexiones sobre la intervención psicológica en emergencias: el caso español. Cuadernos de Crisis, 2(2), 31-35.

McNally, R. J., Bryant, R. A., \& Ehlers, A. (2003). Does early psychological intervention promote recovery from posttraumatic stress? Psychological Science in the Public Interest, 4(2), 46-79.

Martín, L., \& Muñoz, M. (2009). Primeros auxilios psicológicos. Madri: Editorial Sintesis.

Maslow, A. (1954). Religions, values, and peakexperiences. Columbus, $\mathrm{OH}$ : Ohio State University Press. 
Ministério da Saúde. (2010). Saúde Brasil, 2009: Uma análise da situação de saúde e da agenda nacional e internacional de prioridades em saúde. Brasília, DF: Ministério da Saúde.

Molina, R. (2011). A psicologia das emergências e desastres e compromisso social: a experiência latino-americana. In: Conselho Federal de Psicologia (Eds.), Psicologia de emergências e desastres na América Latina: promoção de direitos e construção de estratégias de atuação (pp. 87-94). Brasília: Conselho Federal de Psicologia.

National Child Traumatic Stress Network, National Center for Posttraumatic Stress Disorder (2006). Psychological first aid: field operations guide (2a ed.). Los Angeles: National Child Traumatic Stress Network.

North, C. S., Hong, B. A., Suris, A., \& Spitznagel, E. L. (2008). Distinguishing distress and psychopathology among survivors of the Oakland/Berkeley firestorm. Psychiatry, $71(1), 35-45$.

Organización Panamericana de la Salud [OPAS]. (2004). Manual de evaluación de daños y necesidades en salud para situaciones de desastre. Equador: Organización Panamericana de la Salud/Organización Mundial de la Salud.

Organización Panamericana de la Salud [OPAS]. (2006). Guía práctica de salud mental en situaciones de desastres. Washington: Organización Panamericana de la Salud.

Organización Panamericana de la Salud [OPAS]. (2010). Apoyo psicosocial en emergencias humanitarias y desastres. Washington: Organización Panamericana de la Salud.

Paludo, S. S., \& Koller, S. H. (2007). Psicologia positiva: uma nova abordagem para antigas questões. Paidéia, 17(36), 9-20.

Panagioti, M., Gooding, P., Tarrier, N. (2009). Post-traumatic stress disorder and suicidal behavior: A narrative review. Clinical Psychology Review, 29(6), 471-82.

Quarantelli, E. L. (1954). The nature and conditions of panic. American Journal of Sociology, 60(3), 267-275
Robles, J. I., \& Medina, J. L. (2002). Intervención psicológica en las catástrofes. Madri: Editorial Sintesis.

Rogers, C. R. (1959). A theory of therapy, personality, and interpersonal relationships, as developed in the client-centered framework. In S. Koch (Ed.), Psychology: A study of a science: Formulations of the person and the social context (pp. 184256). New York, NY: McGraw-Hill.

Rogers, C. R. (1961). On becoming a person. Boston, MA: Houghton Mifflin.

Rubio, L. R. (2011). Desastres y vida cotidiana: algunas consideraciones desde la psicología. Cuadernos de Crisis, 1(1), 26-32.

Seligman, M. (2002). Positive psychology, positive prevention, and positive therapy. In C. R. Snyder, \& S. J. Lopez (Eds.), Handbook of positive psychology (pp. 3-9). New York, NY: Oxford University Press.

Seligman, M., \& Csikszentmihalyi, M. (2000). Positive psychology: An introduction. American Psychologist, 55, 5-14.

Slaikeu, K. A. (1996). Intervención en crisis: Manual para práctica e investigación. México: Manual Moderno.

Snyder, C. R., \& Lopez, S. J. (2009). Psicologia positiva: Uma abordagem científica e prática das qualidades humanas. Porto Alegre: Artmed.

Taylor, A. J., \& Frazer, A. G. (1982). The stress of post disaster body handling and victim identification work. Journal Human Stress, 8(4), 4-12.

Valero, S. (2001). Psicología en emergencias y desastres una nueva especialidad. Recuperado em 21 de julho de 2012, de http://www.momografias.com/trabajos10/ emde/emde.shtml

Wainrib, B. R., \& Bloch, E. L. (2000). Intervención en crisis y respuesta al trauma: Teoría y práctica. Bilbao: Desclée de Brouwer.

World Health Organization [WHO]. (2011). Psychological first aid: Guide for field workers. Washington, DC: World Health Organization.

Yunes, M. A. M. (2003). Psicologia positiva e resiliência: $O$ foco no indivíduo e na família. Psicologia em Estudo, Maringá, 8(esp.), 75-84. 